\title{
Prevalência de aneurismas do ventrículo esquerdo em pacientes chagásicos crônicos de duas áreas do Estado do Piauí
}

\author{
Prevalence of left ventricular aneurysms among chronic Chagas \\ disease patients from two areas in the State of Piauí, Brazil
}

\author{
José Borges-Pereira ${ }^{1}$, Sérgio Salles Xavier ${ }^{2,4}$, Andréa Silvestre de Sousa ${ }^{2}$, \\ José Adail Fonseca de Castro ${ }^{3}$, Patrícia Lago Zauza ${ }^{1}$ e José Rodrigues Coura ${ }^{1}$
}

\begin{abstract}
RESUMO
Para avaliar a prevalência de aneurismas do ventrículo esquerdo, realizou-se um estudo transversal envolvendo uma coorte de 261 pacientes chagásicos crônicos (156 do município de João Costa e 105 de São do João do Piauí; 146 mulheres e 115 homens com idade média de $57,9 \pm 14,2$ anos). A avaliação cardiológica foi realizada por exame clinico, eletrocardiograma de repouso e ecocardiograma uni e bidimensional. Foram diagnosticados 23 (8,8\%) aneurismas do ventrículo esquerdo: 17 (6,5\%) no ápice, quatro (1,5\%) na parede posterior, um (0,4\%) na parede inferior e um $(0,4 \%)$ no septo interventricular. Cinco (1,9\%) pacientes de São João do Piaui apresentaram trombos associados com aneurismas apicais. A prevalência de aneurismas do ventrículo esquerdo foi maior entre os pacientes de São João do Piauí (13,3\%) e entre os homens (13,9\%), com diferença não significativa em função da faixa etária. Esses resultados revelam baixa prevalência de aneurismas do ventrículo esquerdo entre os pacientes de João Costa e elevada concentração de trombos entre os pacientes de São João do Piauí.
\end{abstract}

Palavras-chaves: Doença de Chagas. Cardiopatia chagásica crônica. Aneurismas do ventrículo esquerdo. Aneurisma apical. Estado do Piauí.

\begin{abstract}
A cross-sectional study involving a cohort of 261 chronic Chagas disease patients (156 from the municipality ofJoão Costa and 105 from São João do Piaui; 146 women and 115 men with mean age of $57.9 \pm 14.2$ years) was carried out to evaluate the prevalence of left ventricle aneurysms. The cardiological evaluation was performed by means of clinical examination, resting electrocardiograms and one and two-dimensional echocardiograms. A total of 23 cases of left ventricle aneurysms (8.8\%) were diagnosed: 17 (6.5\%) in the apical segment, four (1.5\%) in the posterior wall, one (0.4\%) in the inferior wall and one (0.4\%) in the interventricular septum. Five patients (1.9\%) from SãoJoão do Piaui presented thrombi associated with apical aneurysms. The prevalence of left ventricle aneurysms was greater among the patients from São João do Piauí (13.3\%) and among men (13.9\%), with no significant difference in relation to age group. These results show that there was low prevalence of left ventricle aneurysms among patients from João Costa and high concentration of thrombi among patients from São João do Piauí.
\end{abstract}

Key-words: Chagas disease. Chronic Chagas cardiopathy. Left ventricular aneurysms. Apical aneurysm. State of Piauí.

A doença de Chagas, descrita em 1909 por Carlos Chagas entre moradores de Lassance, Minas Gerais, Brasil ${ }^{14}$, apresentase como importante problema de saúde pública na América Latina $^{24}$, diante da magnitude da cardiopatia, responsável por elevada taxa de absenteísmo e mortalidade, especialmente na população rural ${ }^{18}$. A cardiopatia chagásica crônica (CCC) ao longo de quase um século de estudos tem sido caracterizada por marcadores clínico-evolutivos do tipo bloqueio completo do ramo direito (BRD III) associado ao hemibloqueio anterior esquerdo
(HBAE), extra-sístoles ventriculares polimórficas e aneurismas do ventrículo esquerdo (ANVE), em especial da região apical. 0 aneurisma apical, descrito por Chagas em $1916^{15}$ entre chagásicos brasileiros, tem sido diagnosticado em chagásicos da Venezuela ${ }^{1}$ e da Argentina ${ }^{32}$, e apresenta-se como lesão de elevado potencial gerador de graves distúrbios eletrofuncionais e tromboembolismo ${ }^{26}$ ${ }^{283336}$. No Brasil, a maioria dos estudos sobre a prevalência de ANVE na doença de Chagas, em especial da região apical, origina-se de coortes selecionadas por sintomas em hospitais terciários ou em

\footnotetext{
1. Laboratório de Doenças Parasitárias, Instituto Oswaldo Cruz, Fundação Oswaldo Cruz, Rio de Janeiro, RJ. 2. Instituto de Pesquisas Clínicas Evandro Chagas, Fundação Oswaldo Cruz, Rio de Janeiro, RJ. 3. Faculdade de Ciências Médicas, Universidade do Estado do Piauí, Teresina, PI. 4. Serviço de Cardiologia do Hospital Universitário Clementino Fraga Filho, Universidade Federal do Rio de Janeiro, Rio de Janeiro, RJ.

Apoio da Secretaria de Saúde do Estado do Piauí e Fundação Oswaldo Cruz

Endereço para correspondência: Dr. José Borges-Pereira. Laboratório de Doenças Parasitárias/Dept ${ }^{\circ}$ de Medicina Tropical/FIOCRUZ. Av. Brasil 4365, Pavilhão Arthur Neiva, sala 17, Manguinhos, 21045-900 Rio de Janeiro, RJ.

Tel: $55212280-3740$

e-mail: borges@ioc.fiocruz.br

Recebido para publicação em: 26/07/2007

Aceito em: 19/09/2007
} 
ambulatórios de referência ${ }^{31011} 122934$, enquanto a prevalência de ANVE em população rural de área endêmica, até o momento, foi avaliada através de poucos estudos ${ }^{935}$.

No Estado do Piauí, local do presente estudo, a CCC tem sido avaliada através de estudos epidemiológicos pontuais, empregando o exame clínico e eletrocardiogramas de repouso (ECG). Os primeiros casos de CCC foram descritos por Figueiredo e cols ${ }^{20}$ em pacientes de Oeiras, Castelo e Bom Jesus de Gurguéia. Em seguida, Correia-Lima ${ }^{16}$ e Coura e cols ${ }^{17}$ encontraram 40,3\% de ECG alterados em chagásicos crônicos das localidades de Colônia e Oitis no município de Oeiras; Alves-Filho, em $1995^{4}$, na mesma área, registrou 42,5\% de ECG alterados em chagásicos crônicos e Borges-Pereira e cols ${ }^{7}$ registraram 41,3\% de ECG alterados em chagásicos crônicos de João Costa. $\mathrm{Na}$ literatura, não há referências de estudos sobre ANVE na população chagásica do Estado do Piauí, principal motivo para realizarmos o presente estudo transversal, avaliando-se as características dos ANVE em uma coorte de chagásicos crônicos dos municípios de João Costa e São João do Piauí, área com elevada prevalência de pessoas infectadas pelo Trypanosoma cruzi ${ }^{8}$.

\section{MATERIAL E MÉTODOS}

Área de estudo. Constitui-se dos municípios de João Costa e São João do PI, localizados no Sudeste do Estado do Piauí (PI), região do semi-árido brasileiro, com superfície total de $3.248 \mathrm{~km}^{2}$, altitude média de $300 \mathrm{~m}$; temperatura média anual de $24^{\circ} \mathrm{C}$ e $26^{\circ} \mathrm{C}$ (máxima de $42^{\circ} \mathrm{C}$ e $8^{\circ} \mathrm{C}$ de mínima), vegetação do tipo Caatinga, a 382Km de Teresina (Figura 1). De acordo com o Instituto Brasileiro de Geografia e Estatística (IBGE) ${ }^{22}$, a população dos dois municípios está estimada em 20.295 habitantes, com índice de desenvolvimento humano (IDH) de 0,654. Para a área de estudo, os dados dos inquéritos sorológico e entomológico realizados em 2002 por Borges-Pereira e cols ${ }^{8}$ revelaram prevalência da infecção chagásica em $6 \%$ da população rural, com queda significativa nos últimos 30 anos, eliminação do Triatoma infestans e redução da densidade dos triatomíneos do peridomicílio com provável interrupção da transmissão vetorial. Cabe destacar que o município de João Costa era território de São João do Piauí até 1997 e que nos inquéritos sorológico e entomológico nacionais realizados no período 1975-1980, São João do Piauí apresentava Triatoma infestans em domicílios e uma soroprevalência de $28,9 \%$ na população rural.

Coorte. Incluiu 261 pacientes, sendo 156 de João Costa e 105 de São João do Piauí, 146 mulheres e 115 homens com idade média de 57,9 \pm 14,2 anos, todos soropositivos para IgG anti-Trypanosoma cruzi pelos testes de imunofluorescência indireta (produzido por Bio-Manguinhos) e ELISA com antígenos recombinantes (produzido por Wienner) e originários dos inquéritos sorológicos realizados nesses municípios entre 1999 e 2002. Para evidenciar a cardiopatia e aneurismas do ventrículo esquerdo, em abril de 2007, os pacientes foram avaliados por exame clínico, eletrocardiograma de repouso e ecocardiograma bidimensional.

Eletrocardiograma. Após o exame clínico dirigido ao aparelho cardiovascular, utilizou-se um eletrocardiógrafo digital marca Micromed biotecnologia e obteve-se o registro das doze derivações clássicas (periféricas e precordiais) e DII longo em caso de arritmias, realizando-se a leitura de acordo com os critérios da New York Heart Association ${ }^{25}$, considerandose normais frequiências de 60 a 120 batimentos por minuto e arritmias sinusais.

Ecocardiograma. Na avaliação ecocardiográfica utilizou-se um ecocardiógrafo portátil marca Acuson, modelo Cypress, com imagem na freqüência fundamental e em harmônica de tecido. Todos os ecocardiogramas foram realizados por um dos autores do estudo (Xavier SS), sem prévio conhecimento da avaliação clínica e eletrocardiográfica. 0 exame incluiu cortes convencionais (para e supra esternais, apicais e subcostais) e variações dos cortes convencionais, principalmente dos apicais, com objetivo de identificar alterações segmentares localizadas (geralmente pequenos aneurismas mamilares). A função sistólica global do ventrículo esquerdo (VE) foi avaliada a modo $\mathrm{M}$, através do cálculo da fração de ejeção (FE) segundo Teicholz e cols ${ }^{31}$. Devido ao caráter freqüentemente segmentar da cardiopatia chagásica crônica, a função sistólica global do VE foi também avaliada ao bidimensional, de forma subjetiva, sendo classificada em normal

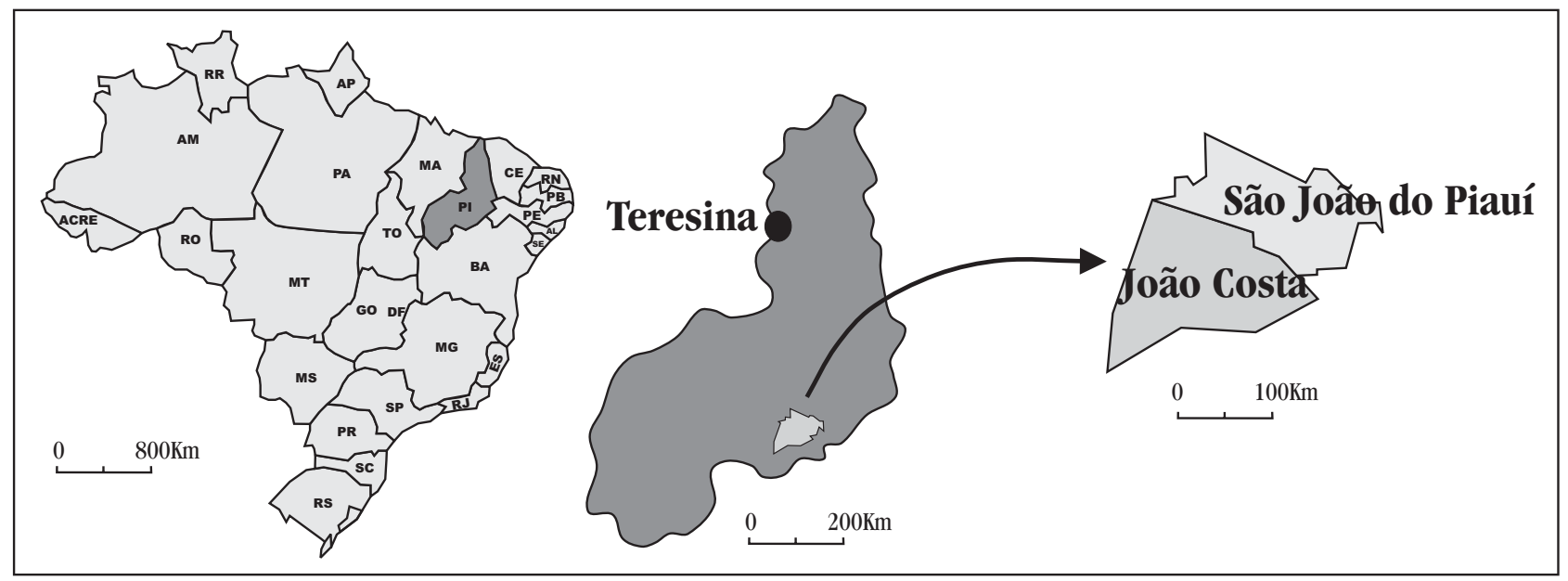

Figura 1 - Área de estudo: São João do Piauí e João Costa. 
( $\mathrm{FE} \geq 55 \%)$, levemente $(\mathrm{FE} \geq 45 \%$ e $<55 \%)$, moderadamente (FE $\geq 35 \%$ e $<45 \%$ ) e gravemente deprimida ( $\mathrm{FE}<35 \%$ ). A estimativa da FE do VE através de análise subjetiva foi validada por estudo prévio ${ }^{5}$, no qual se demonstrou boa correlação com a medida realizada por ventriculografia radioisotópica, desde que realizada por ecocardiografista experiente. Na análise segmentar utilizou-se o modelo de 17 segmentos preconizado atualmente pela Sociedade Americana de Ecocardiografia ${ }^{30}$. Diagnosticou-se aneurisma ventricular na presença de deformidade para fora da linha endocárdica, persistindo em sístole e diástole ${ }^{10}$.

Ética e estatística. 0 trabalho, parte do projeto sobre o perfil epidemiológico da doença de Chagas na Caatinga brasileira, foi aprovado pelo Comitê de Ética de Pesquisas em Seres Humanos da Fundação Oswaldo Cruz (FIOCRUZ), protocolo 289/05, e as pessoas adultas e responsáveis por menores assinaram o Termo de Consentimento Informado Livre e Esclarecido, conforme a Resolução 196/1996 do Conselho Nacional de Saúde, participando espontaneamente após esclarecimentos dos seus riscos e benefícios. Utilizando-se os programas Excel e Epi-Info 6.04, construiu-se um banco de dados com aspectos demográficos, eletrocardiográficos e ecocardiográficos. Na comparação das variáveis categóricas realizou-se análise univariada, utilizandose o teste do qui-quadrado ou o teste exato de Fischer e, na comparação das médias, utilizou-se à análise de variância (ANOVA), considerando-se o nível de significância de 5\%.

\section{RESULTADOS}

$\mathrm{Na}$ avaliação eletrocardiográfica, 108 (41,4\%) pacientes apresentaram ECG alterado, sendo 64 (41\%) procedentes de João Costa e $44(41,9 \%)$ de São João do Piauí (p > 0,05). Em relação ao gênero, 59 (51,3\%) homens e 49 (33,6\%) mulheres apresentaram ECG alterado $\left(X^{2}=8,35 ; g l=1 ; p=0,0038\right)$. Em relação à faixa etária (Tabela 1 ), houve aumento da frequiência de ECG alterados com o aumento da idade $\left(\mathrm{X}^{2}=23,03\right.$; $\mathrm{gl}=4 ; \mathrm{p}=0,0001)$. Entre as alterações, distribuídas na Tabela 2, predominaram, de modo significativo, o BRD III associado ao HBAE em $16 \%$ dos pacientes.

$\mathrm{Na}$ avaliação ecocardiográfica, 31 (11,9\%) pacientes apresentaram exame alterado, sendo que $23(8,8 \%)$ apresentaram aneurismas do ventrículo esquerdo, 17 (6,5\%) no ápice, quatro $(1,5 \%)$ na parede posterior, um $(0,4 \%)$ na parede inferior e um $(0,4 \%)$ no septo interventricular. Considerando-se os resultados em relação à população de cada município, foram diagnosticados 14 (13,3\%) ANVE entre os 105 pacientes de São João do PI, todos na região apical, sendo cinco com trombos e nove $(5,8 \%)$ ANVE entre os 156 pacientes de João Costa $\left(X^{2}=4,47 ; \mathrm{gl}=1\right.$; $\mathrm{RR}=2,3 ; \mathrm{p}=0,034)$ : sendo quatro na parede posterior, três no ápice, um na parede inferior e um no septo interventricular. Em relação ao gênero, foram diagnosticados $16(13,9 \%)$ aneurismas entre os homens e sete $(4,8 \%)$ entre as mulheres $\left(X^{2}=6,66\right.$; $\mathrm{gl}=1 ; \mathrm{RR}=2,9 ; \mathrm{p}=0,009)$. Em relação à faixa etária (Tabela 1), a ocorrência de aneurismas não se mostrou associada à idade $\left(\mathrm{X}^{2}=0,160 ; \mathrm{gl}=4 ; \mathrm{p}=0,688\right)$. A idade média dos pacientes com aneurismas foi de $57 \pm 12,5$ anos, enquanto a idade média dos pacientes sem aneurismas foi de $58 \pm 14,4$ anos $(\mathrm{p}>0,05)$.

0 grave comprometimento da função ventricular foi diagnosticado em sete $(2,7 \%)$ pacientes, todos portadores de ANVE, sendo dois com trombos. A fração de ejeção média da coorte foi de $66 \% \pm 10 \%$, sem diferença significativa em relação ao gênero e a faixa etária. Todavia, a fração de ejeção média dos pacientes com aneurismas $(49,7 \% \pm 16,6 \%)$ foi significativamente inferior a do grupo de pacientes sem aneurismas $(67,7 \% \pm 7,9 \%)$ $(\mathrm{p}=0,02)$. Todos os pacientes com aneurismas apresentavam ECG alterados (Tabela 3). Entre os 14 pacientes com aneurisma apical, de São João do Piauí, cinco apresentavam trombos (Figura 2), enquanto entre os pacientes de João Costa com ANVE não houve diagnóstico de trombos.

\begin{tabular}{|c|c|c|c|c|c|}
\hline Variáveis & Pacientes & $\mathrm{ECGa}$ & $\mathrm{ECGa}(\%)$ & ANVE & ANVE (\%) \\
\hline \multicolumn{6}{|l|}{ Município de origem } \\
\hline João Costa & 156 & 64 & 41,0 & 9 & 5,8 \\
\hline $\begin{array}{l}\text { São João do Piauí } \\
\text { análise estatística }\end{array}$ & 105 & 44 & $\begin{array}{c}41,9 \\
X^{2}=0,02 ; p=0,887\end{array}$ & 14 & $\begin{array}{c}13,3 \\
X^{2}=4,47 ; p=0,034\end{array}$ \\
\hline \multicolumn{6}{|l|}{ Sexo } \\
\hline masculino & 115 & 59 & 51,3 & 16 & 13,9 \\
\hline $\begin{array}{l}\text { feminino } \\
\text { análise estatística }\end{array}$ & 146 & 49 & $\begin{array}{c}33,6 \\
X^{2}=8,35 ; p=0,0038\end{array}$ & 7 & $\begin{array}{c}4,8 \\
X^{2}=6,66 ; p=0,009\end{array}$ \\
\hline \multicolumn{6}{|l|}{ Faixa etária (anos) } \\
\hline $18-29$ & 11 & 1 & 9,1 & 1 & 9,1 \\
\hline $30-39$ & 14 & 9 & 64,3 & 2 & 14,3 \\
\hline $40-49$ & 46 & 8 & 17,4 & 2 & 4,4 \\
\hline $50-59$ & 72 & 30 & 41,7 & 6 & 8,3 \\
\hline $\begin{array}{l}\quad \geq 60 \\
\text { análise estatística }\end{array}$ & 118 & 60 & $\begin{array}{c}50,8 \\
X^{2}=23,03 ; p=0,0001\end{array}$ & 12 & $\begin{array}{c}10,2 \\
X^{2}=0,160 ; p=0,688\end{array}$ \\
\hline
\end{tabular}


Tabela 2 - Freqüiência das alterações eletrocardiográficas em 261 pacientes chagásicos crônicos de João Costa e São João do Piauí, Brasil, 2007.

\begin{tabular}{lcc}
\hline Alterações eletrocardiográficas & Número & Percentagem \\
\hline Bloqueio do ramo direito grau III (BRD III) & 52 & 19,9 \\
Hemibloqueio anterior esquerdo (HBAE) & 47 & 18,0 \\
Bloqueio átrio-ventricular grau I (BAV I) & 18 & 6,9 \\
Extra-sístoles ventriculares monomórficas (EV m) & 16 & 6,1 \\
Alteração primária da repolarização ventricular (APRV) & 15 & 5,7 \\
Zona eletricamente inativa (ZEI) & 13 & 4,9 \\
Alteração difusa da repolarização ventricular (ADRV) & 6 & 2,3 \\
Bradicardia sinusal (BS) & 4 & 1,5 \\
Sobrecarga atrial esquerda (SAE) & 4 & 1,5 \\
Bloqueio do ramo esquerdo grau III (BRE III) & 4 & 1,5 \\
Extra-sístoles supraventriculares (ESV) & 3 & 1,3 \\
Sobrecarga ventricular esquerda (SVE) & 3 & 1,3 \\
Extra-sístoles ventriculares polimórficas (EV p) & 2 & 0,8 \\
Baixa voltagem do complexo QRS (BV-QRS) & 1 & 0,4 \\
Bloqueio átrio-ventricular grau II (BAV II) & 1 & 0,4 \\
\hline
\end{tabular}

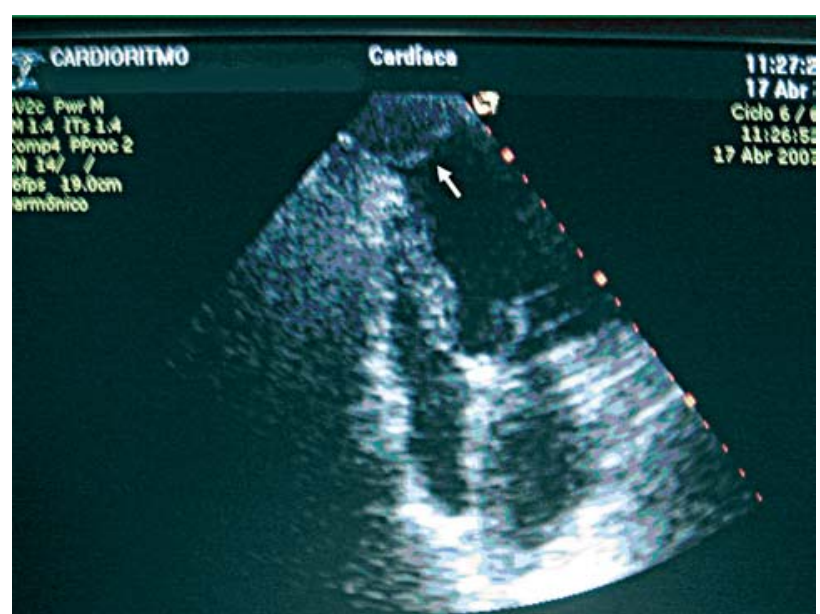

Figura 2 - Aneursima apical preenchido por volumoso trombo (seta), melhor visível neste corte apical modificado.

\section{DISCUSSÃo}

Desenvolver pesquisa de campo sobre a doença de Chagas em populações de zonas rurais de áreas endêmicas se constitui em enorme desafio para os profissionais da área de saúde. Identificam-se os agravos e estabelecem-se planos de controle e tratamento que, em geral, esbarram nas precárias condições dos serviços de assistência as populações. Nosso grupo, formado por médicos e pesquisadores da Fundação Oswaldo Cruz/RJ e da Universidade Federal do Rio de Janeiro, tem realizado pesquisas de campo, em diferentes áreas endêmicas do Brasil, sobre a cardiopatia chagásica crônica, com destaque para os aneurismas do ventrículo esquerdo, na tentativa de identificar possíveis diferenças regionais na epidemiologia e clínica da doença de Chagas. Neste contexto, em 1995, realizou-se o primeiro estudo ecocardiográfico de campo no município de Virgem da Lapa, MG, avaliando-se uma coorte de 298 pacientes chagásicos crônicos não selecionados por sintomas, identificando-se $42 \%$ dos pacientes com ECG alterados, 18,8\% com aneurismas do ventrículo esquerdo e 15\% com aneurisma apical'. Em 2004, no Estado do Amazonas, realizou-se o segundo estudo, avaliandose a cardiopatia em uma série de 110 pacientes com história epidemiológica de exposição a triatomíneos em piaçabais ao longo
Tabela 3 - Características dos pacientes chagásicos crônicos com aneurismas do ventrículo esquerdo, Estado do Piauí, Brasil, 2007.

\begin{tabular}{lccccc}
\hline Paciente & Sexo & $\begin{array}{c}\text { Idade } \\
\text { (anos) }\end{array}$ & Eletrocardiograma & $\begin{array}{c}\text { Fração de } \\
\text { ejeção }\end{array}$ & ANVE \\
\hline JC 0067 & M & 31 & BRD III, HBAE & 63 & apical \\
JC 0316 & M & 74 & BAV I & 21 & parede posterior \\
JC 0467 & M & 66 & BAV I, APRV & 46 & apical \\
JC 0803 & M & 61 & BS & 56 & parede posterior \\
JC 0821 & M & 68 & BRD III & 52 & parede inferior \\
JC 0981 & F & 62 & APRV & 37 & parede posterior \\
JC 2025 & M & 67 & BRD III, HBAE, EV & 36 & parede posterior \\
JC 2027 & F & 67 & BRD III, HBAE, EV & 76 & apical \\
JC 2152 & F & 38 & BRE III & 28 & septal \\
SJ 0472 & M & 50 & BRD III, HBAE & 70 & apical \\
SJ 0489 & M & 52 & BRD III, ZEI & 40 & apical \\
SJ 0506 & M & 62 & BRD III, HBAE, ZEI & 68 & apical \\
SJ 0541 & M & 49 & BAV I, EVP & 44 & apical \\
SJ 0552 & F & 28 & APRV & 58 & apical \\
SJ 0571 & M & 62 & BRD III, HBAE, EV, ZEI & 46 & apical* \\
SJ 0575 & F & 67 & BRD III, ZEI & 46 & apical \\
SJ 0576 & F & 66 & BRE III & 35 & apical* \\
SJ 0805 & F & 46 & ZEI & 49 & apical* \\
SJ 0812 & M & 57 & APRV, HBAE & 64 & apical \\
SJ 0814 & M & 54 & APRV & 68 & apical \\
SJ 0815 & M & 53 & APRV & 47 & apical* \\
SJ 1002 & M & 52 & ADRV, SVE, ZEI & 25 & apical* \\
SJ 1012 & M & 74 & APRV & 69 & apical \\
\hline
\end{tabular}

* presença de trombos JC: João Costa SJ: São João do Piaúí BAV I: bloqueio átrioventricular grau I: BRE III: bloqueio do ramo esquerdo grau III BRD III: bloqueio do ramo direito grau III HBAE: hemibloqueio anterior esquerdo EV: extra-sístoles ventriculares monomórficas APRV: alteração primária da repolarização ventricular BS: bradicardia sinusal ADRV: alteração difusa da repolarização ventricular SVE: sobrecargaventricular esquerdaZEI:zona eletricamente inativaEVp: extra-sístoles ventriculares polimórficas.

do Rio Negro e seus afluentes e/ou pacientes com sintomatologia sugestiva de insuficiência cardíaca, diagnosticando-se três pacientes com aneurisma apical ${ }^{35}$. 0 presente estudo é o terceiro realizado pelo grupo e o primeiro realizado no Estado do Piaú, avaliando-se uma coorte de 261 pacientes não selecionados por sintomas, diagnosticando-se $41,4 \%$ de pacientes com ECG alterados, 8,8\% com aneurismas do ventrículo esquerdo, 6,9\% com aneurismas apicais e 1,9\% com trombos.

Ao considerarmos os aneurismas do ventrículo esquerdo, representados principalmente pelos aneurismas apicais, como marcadores de gravidade da cardiopatia chagásica crônica, os resultados dos estudos realizados pelo nosso grupo configuram maior gravidade da CCC na coorte de pacientes de Virgem da Lapa-MG em comparação com a presente coorte de pacientes do Piauí. Essa diferença na gravidade pode ser validada, de maneira indireta, pela prevalência de extra-sístoles ventriculares em 16\% dos pacientes da coorte de Virgem da Lapa, MG e em 7\% dos pacientes da presente coorte do Piauí.

Do mesmo modo que na maioria dos estudos sobre a doença de Chagas, em diferentes coortes e regiões, no presente estudo foi identificada maior frequiência de aneurisma apical em comparação com os demais tipos de aneurismas do ventrículo esquerdo. Até 0 momento, não há explicação definida para gênese do aneurisma apical. No campo das hipóteses, atribui-se a participação de múltiplos fatores, entre os quais o comprometimento do sistema de condução, produzindo retardo na ativação da área da ponta do coração, resultando em elevada distensão regional que evoluiria 
para a formação do aneurisma ${ }^{6}$ e a indicação de uma fase vascular interveniente na patogênese do aneurisma apical ${ }^{27}$.

A literatura revela amplo intervalo de valores na prevalência de aneurisma apical em coortes de pacientes chagásicos, valores dependentes de características das coortes e da tecnologia empregada na avaliação da lesão. Há valores de $30 \%$ a $86,9 \%$ observados em estudos de necropsias ${ }^{1323}$, de $40 \%$ a $77 \%$ identificados por ventriculografia ${ }^{21}$ e de $14 \%$ a $46 \%$ diagnosticados por ecocardiograma ${ }^{1035}$.

O grupo de pacientes de São João do Piauí apresentou maior prevalência de ANVE do que o grupo de pacientes de João Costa, enquanto ambos os grupos de pacientes apresentaram prevalências semelhantes de ECG alterados (Tabela 1). Essas observações, além de revelarem a significativa diferença regional na gravidade da cardiopatia entre os pacientes dos dois municípios estudados, expressam que o ecocardiograma tem maior resolução na definição clínico-funcional do que o eletrocardiograma.

$\mathrm{Na}$ presente coorte, os homens apresentaram maior prevalência de ECG alterado do que as mulheres, à semelhança com os resultados da maioria dos estudos dessa natureza ${ }^{7} 16$, porém, a maior prevalência de ANVE entre os homens difere do resultado na coorte de Virgem da Lapa, na qual a prevalência não esteve associada ao gênero. Essa diferença regional pode estar relacionada principalmente com as características das coortes. A maior prevalência de cardiopatia chagásica crônica entre os homens, até o momento não tem explicação definida, a partir de estudos analíticos controlados que possam evidenciar causas. Por outro lado, os estudos descritivos mostram forte associação entre a prevalência e gravidade da cardiopatia chagásica crônica e o sexo masculino com os autores, em hipótese, atribuindo responsabilidade, principalmente à atividade física.

Na coorte do presente estudo e na de Virgem da Lapa/MG ${ }^{9}$, a prevalência de ANVE e da região apical não variou com a idade dos pacientes, enquanto a prevalência de cardiopatia, com base no resultado do ECG, aumentou com a idade. Essas observações indicam que o desenvolvimento da miocardiopatia chagásica crônica depende do tempo de evolução da infecção pelo Trypanosoma cruzi, enquanto o desenvolvimento do aneurisma apical independe do tempo de evolução. Entre os diversos fatores que podem estar relacionados com a gênese do aneurisma apical independentemente do tempo de evolução da doença está o parasitismo tissular que pode cursar com multiplicação do parasito, ruptura celular, liberação das formas circulantes, produzindo parasitemia patente, reação inflamatória, lesão tecidual e fibrose.

Os ANVE, principalmente da região apical, marcadores da CCC, são diagnosticados predominantemente entre pacientes com ECG alterado, podendo ser diagnosticados, com baixa frequiência, em pacientes assintomáticos e com ECG normal ${ }^{32} 33$. Na presente coorte, todos os aneurismas foram diagnosticados em pacientes com ECG alterado. Entre as alterações associadas com ANVE estão aquelas consideradas marcadoras da CCC como BRD III associado ao HBAE e extra-sístoles ventriculares polimórficas. Ao considerarmos os ANVE associados as extra-sístoles ventriculares, a prevalência dessas arritmias pode ser indicadora da prevalência de ANVE em uma determinada população. Como exemplo, na coorte de Virgem da Lapa/MG foram diagnosticados 16,9\% de extra-sístoles ventriculares e 18,8\% de ANVE, enquanto na coorte do presente estudo foram diagnosticados, respectivamente, $6,9 \%$ e 8,8\%. Por outro lado, a análise especifica sobre a associação entre extra-sístoles ventriculares e ANVE na coorte de Virgem da Lapa examinada em $1995^{\circ}$ revelou a presença de extra-sístoles ventriculares em $37(66,1 \%)$ dos 56 pacientes com ANVE, em $10(66,7 \%)$ dos 15 pacientes com acinesias e hipocinesias e em 40 (32\%) dos 125 pacientes com cardiopatia sem ANVE, configurando-se uma associação direta entre as frequiências de extra-sístoles ventriculares e de ANVE $\left(X^{2}=18,37 ; p=0,00001\right)$, enquanto na coorte do presente estudo, os percentuais de extrasístoles ventriculares foram de 17,4\% (4/23) entre os pacientes com ANVE e de 16,5\% (14/85) entre os pacientes com cardiopatia sem ANVE, valores significativamente menores do que os encontrados na coorte de Virgem da Lapa, marcando importante diferença regional na gravidade da CCC nessas áreas.

A presença de trombos associados ao aneurisma apical em pacientes chagásicos tem sido bastante assinalada em estudos referidos na literatura ${ }^{26}$. Estudos de autópsia ou de natureza longitudinal revelam a elevada incidência de eventos tromboembólicos, com aumento significativo da perspectiva de morte por acidente vascular encefálico. Fernandes e cols ${ }^{19}$, estudando a frequiência de trombose apical em corações de chagásicos crônicos, encontraram maior percentual no grupo de pacientes com lesão apical sem afastamento dos feixes musculares em comparação com o grupo de pacientes com lesão apical com afastamento dos feixes musculares. 0 diagnóstico de trombos no ventrículo esquerdo em cinco pacientes de São João do Piauí motivou, de imediato, uma reunião do nosso grupo com a comunidade médica da região, na qual foram apresentadas as seguintes demandas: os pacientes portadores de trombos, o imediato tratamento com anticoagulante e as técnicas laboratoriais imprescindiveis para o controle desse tipo de tratamento.

Em suma, os resultados do presente estudo revelaram que a doença de Chagas nos municípios de João Costa e São João do Piauí se apresenta com índice de cardiopatia chagásica crônica semelhante ao de Virgem da Lapa/MG, porém com menor gravidade, configurada pela menor prevalência de aneurismas do ventrículo esquerdo, principalmente da região apical.

\section{AGRADECIMENTOS}

Agradecemos aos colegas da CardioRitmo: Jacob Atié, Washington de Andrade Maciel e Eduardo Machado Andréa pela cessão do ecocardiógrafo para a realização dos exames no campo; ao pessoal da área de saúde dos municípios de João Costa e São João do Piauí, pela colaboração em todas as fases do trabalho; a Otacílio Batista, gerente da vigilância em saúde da SESAPI, pelo fornecimento de automóveis para o deslocamento no campo; a José de Souza Nogueira (FIOCRUZ-RJ) e Francisco das Chagas (SESAPI) pela colaboração na obtenção, armazenamento e acondicionamento dos soros. 


\section{REFERÊNCIAS}

1. Acquatella H, Schiller NB, Puigbó JJ, Giordano H, Suarez JA, Casal H, Arreaza N, Valecillos R, Hirschhaut E. M-mode and two-dimensional echocardiography in chronic Chagas' heart disease. Circulation 62: 787-795, 1980.

2. Albanesi-Filho FM, Gomes-Filho JBM. Acometimento da ponta do ventrículo esquerdo na cardiopatia chagásica crônica: aspectos clínicos e ventriculográficos. Arquivos Brasileiros de Cardiologia 52: 115-120, 1989.

3. Albanesi-Filho FM, Gomes-Filho JBM. A lesão apical do ventrículo esquerdo na evolução clínica da cardiopatia chagásica crônica. Arquivos brasileiros de cardiologia 56: 457-463, 1991

4. Alves Filho FI. Morbidade da doença de Chagas em áreas endêmicas dos municípios de Oeiras e Colônia do Piauí. Dissertação de Mestrado, Instituto Oswaldo Cruz, Fundação Oswaldo Cruz, Rio de Janeiro, RJ, 1995.

5. Amico AF, Lichtenberg GS, Reisner AS, Stone CK, Schwartz RG, Meltzer RS. Superiority of visual versus computerized echocardiographic estimation of radionuclide left ventricular ejection fraction. American Heart Journal 118:1259$1265,1989$.

6. Andrade ZA. Lesão apical do coração na miocardite crônica chagásica. Hospital 50:803-812, 1956

7. Borges-Pereira J, Castro JAF, Campos JHF, Nogueira JS, Zauza PL, Marques P, Cardoso MA, Britto C, Araújo AJG. Estudo da infecção e morbidade da doença de Chagas no município de João Costa - Parque Nacional Serra da Capivara, Piauí, Brasil. Revista da Sociedade Brasileira de Medicina Tropical 35:315-322, 2002

8. Borges-Pereira J, Castro JAF, Silva AR, Zauza PL, Bulhões TP, Gonçalves ME, Almeida ES, Salmito MA, Pereira LRM, Alves-Filho FI, Correia-Lima FG, Coura JR. Soroprevalência da infecção chagásica no Estado do Piauí, 2002. Revista da Sociedade Brasileira de Medicina Tropical 39:530-539, 2006.

9. Borges-Pereira J, Xavier SS, Pirmez C, Coura JR. Doença de Chagas em Virgem da Lapa, Minas Gerais, Brasil. IV. Aspectos clínicos e epidemiológicos do aneurisma ventricular esquerdo. Revista da Sociedade Brasileira de Medicina Tropical 31:457-463, 1998.

10. Camara EJN. Alterações segmentares da contratilidade do ventrículo esquerdo na cardiopatia chagásica com e sem dilatação ventricular. Arquivos Brasileiros de Cardiologia 60:151-155, 1993.

11. Carvalhal S. A lesão apical na cardiopatia chagásica. Arquivos Brasileiros de Cardiologia 25:257-262, 1972.

12. Carvalhal S, Bittencourt LAK, Nogueira EA. A lesão apical na cardiopatia chagásica. Arquivos Brasileiros de Cardiologia 35:171-177, 1980.

13. Carvalhal S, Campos FCM, Portugal O. Alteração do complexo QRS nas derivações precordiais e seu substrato anatômico em pacientes portadores de miocardite chagásica crônica. Revista Paulista de Medicina 45:161-168, 1954.

14. Chagas C. Nova Trypanozomíaze Humana. Estudo sobre a morfologia e o ciclo evolutivo do Schizotrypanum cruzi. n. gen., n. sp. ajente etiológico de nova entidade mórbida do homem. Memórias do Instituto Oswaldo Cruz 1:159-218, 1909.

15. Chagas C. Processos patogênicos da trypanosomiase americana. Memórias do Instituto Oswaldo Cruz 8:7-37, 1916

16. Correia-Lima FG. Doença de Chagas no município de Oeiras, Piauí - estudo seccional nas localidades de Colônia e Oitis. Dissertação de Mestrado, Universidade Federal do Rio de Janeiro, RJ, 1976.

17. Coura JR, Abreu LL, Dubois LEG, Correia-Lima FG, Arruda Jr ER, Willcox HPF. Anunziato N, Petana W. Morbidade da doença de Chagas. II - Estudos seccionais em quatro áreas de campo no Brasil. Memórias do Instituto Oswaldo Cruz 79:101-124, 1984.

18. Dias JCP, Schofield CJ. The Evolution of Chagas Disease (American Trypanosomiasis) Control after 90 Years since Carlos Chagas Discovery. Memórias do Instituto Oswaldo Cruz 94 (supl I): 103-121, 1999.
19. Fernandes SO, Oliveira MAS, Teixeira VPA, Almeida HO. Trombose endocárdica e tipo de lesão vorticilar esquerda em chagásicos crônicos. Arquivos Brasileiros de Cardiologia 48:17-19, 1987.

20. Figueiredo PZ, Correia-Lima FG, Portella-Nunes JN. Doença de Chagas: primeiros casos autóctones no estado do Piauí. Revista da Sociedade Brasileira de Medicina Tropical 9:105-107, 1975.

21. Granzotti JA, Marin Neto JA, Galo Jr L, Manco JC, Rassi A, Amorim. Contribuição ao estudo do aneurisma de ponta na cardiopatia chagásica crônica. Arquivos Brasileiros de Cardiologia 27:477-487, 1974.

22. Instituto Brasileiro de Geografia e Estatística. http://www.ibge.gov.br/ censo2000.

23. Moia B, Rosenbaum MB, Hojman D. Aneurismas ventriculares em la miocarditis cronica chagasica. Revista Argentina de Cardiologia 22:113-150, 1955.

24. Moncavo A. Progress Towards Interruption of Transmission of Chagas Disease. Memórias do instituto Oswaldo Cruz 94 (supl I): 401-404, 1999.

25. New York Heart Association (NYHA). Nomenclature and criteria for diagnosis of disease of the heart and great vessels. $7^{\text {th }}$ edition Little and Brow Company, Boston 1973.

26. Nunes MC, Barbosa MM, Rocha MO. Peculiar aspects of cardiogenic embolism in patients with Chagas' cardiomyopathy: a transthoracic and transesophageal echocardiographic study. Journal of the American Society of Echocardiography 18:761-767, 2005.

27. Oliveira JA. Heart aneurysm in Chagas' disease. Revista do Instituto de Medicina Tropical de São Paulo 40: 301-307, 1998. Disponível em: http://www.scielo. br/scielo.php?script=sci_arttext\&pid=\$0036-46651998000500007

28. Oliveira JSM, Araújo RRC, Navarro MA, Muccillo G. Cardiac trombosis and thromboembolism in chronic Chagas' heart disease. The American Journal of Cardiology 52:147-151, 1983.

29. Ortiz J, Barreto ACP, Matsumoto AY, Mônaco CAF, Ianni B, Marotta RHQ, Mady C, Bellotti G, Pileggi F. Alteração contrátil segmentar na forma indeterminada da doença de Chagas. Estudo ecocardiográfico. Arquivos Brasileiros de Cardiologia 49:217-220, 1987.

30. Schiller NB, Shah PM, Crawtord M, DeMana A, Devereux R, Feigenbaum H Gutfesell H, Tajik AS. Recommendations for quantitation of the left ventricle by two-dimensional echocardiography. The Journal of American Society Echocardiography 2:358-365, 1989.

31. Teicholz LE, Kreuler T, Werman MV. Problems in echocardiographic volume determinations: echocardiographic-angiocardiographic correlations in the presence or absence of asynergy. The American Journal of Cardiology 37:7-12, 1976 .

32. Viotti RJ, Vigliano C, Loucella S, Lococo B, Petti M, Bertocchi G, Ruiz-Vera B, Armenti $\mathrm{H}$. Value of echocardiogrphy for diagnosis and prognosis of chronic Chagas disease cardiomyopathy without heart failure. Heart 90:655-660, 2003.

33. Xavier SS. Estudo longitudinal da morbi-mortalidade cardíaca da doença de Chagas em coorte de um grande centro urbano: análise clínica, eletrocardiográfica, radiológica e ecocardiográfica de 604 casos. Tese de doutorado, Universidade Federal do Rio de Janeiro, RJ, 1999.

34. Xavier SS, Sousa AS, Brasil PEAA, Gabriel FG, Holanda MT, Hasslocher-Moreno A. Aneurisma apical na fase crônica da doença de Chagas: Prevalência e valor prognóstico em uma coorte urbana de 1053 pacientes. Revista da Sociedade de Ecocardiografia do Rio de Janeiro 18:351-356, 2006.

35. Xavier SS, Sousa AS, Viñas PA, Junqueira ACV, Bóia MN, Coura JR. Cardiopatia chagásica crônica no Rio Negro, Estado do Amazonas. Relato de três novos casos autóctones, comprovados por exames sorológicos, clínicos, radiográficos do tórax, eletro e ecocardiográficos. Revista da Sociedade Brasileira de Medicina Tropical 39:211-216, 2006.

36. Xavier SS, Sousa AS, Brasil PEAA, Gabriel FG, Holanda MT, Hasslocher-Moreno A, Garcia MY, Siciliano APRV. Incidência e preditores de morte súbita na cardiopatia chagásica crônica com função sistólica preservada. Revista da Sociedade de Cardiologia do Estado do Rio de Janeiro 18:457-463, 2005. 